\title{
TO THE WAVE NATURE OF ECONOMIC CYCLES
}

\author{
Author : Dmitry CHISTILIN
}

\begin{abstract}
The question of economic cycles' origin is still one of the most actual problems. In inorganic nature the wave process appears as a form of system's existence and as reaction of environment against outside disturbance. Socium as an environment, a separate element of which is a human being, possesses two basic characteristics. It is different values of capability of every separate human being, on one hand, and organization of scarce resources for production and level of consumption of limited benefits, on the other hand. These two characteristics create two opposite trends of production and consumption in condition of growth of population and scarceness of resources during long period of time. These two mentioned trends of opposite directions initiate stationary wave process, which economics defines as economic cycle. Therefore, the property of organic system to initiate wave processes under the influence of outside environment is a necessary condition for supporting dynamic stability of the system in the process of its development, i.e. supporting homeostasis.
\end{abstract}

Key words: economic cycle; non-linear economic dynamics; population growth; economic wave process

JEL classification: N1; A12; E3; E32

\section{Introduction}

This work is dedicated to the problem of origin and nature of economic cycle - one of the most important and the oldest unsettled problem of economy theory. A lot of most famous scientists-economists studied phenomena of economic cycles, economic dynamics and development. Among them are: K. Marx, Wicksell, Mitchell, Tugan-Baranovsky, Gassel, Shcumpeter, Kondratyev, Harrod, Hansen, Aftalion, Clark, Spiethof, Kuznets, Pigou et al. and, according to A. Hansen, this problem had not been solved till the time of his work (40s of the $20^{\text {th }}$ century) (Hansen, 1997). By analyzing the works of economists of the second half of the $20^{\text {th }}$ century, it is possible to say that his statement remains true until now. Methodology that exists today in economics and its sections - theory of economic dynamics and theory of economic development - has come from classical mechanics [Kondratyev, 1998; Schumpeter, 1982). Today is a time of complexities. It appears that the organization of universal system is complicated and functions according to non-linear laws. The idea of complexity itself became the focus of scientific thought. Non-linear physics and physics of open systems have occupied their place in educational and research processes of physics (Zaslavsky and Sagdeev, 1988; Klymantovich, 1999; Bar Yam, 1997). The concept of self-organization of physical objects is confirmed as open dissipation systems. Fundamental categories of physics are newly interpreted from the position of new knowledge of complexity. The same phenomenon can be observed in economics as well. Wide diffusion of knowledge, including methods, occurs among different branches of science. Interdisciplinarity of research is one of the fastest spreading scientific methods. Our work is inter-disciplinary. It is an attempt to consider economic problem from position of non-linear physics, or, more exactly, - from position of physics of non-linear wave processes. So we are aware that "pure" physicists interested in economics can reveal triviality and even dilettantism in stated context and they will be right. Economists, acquainted with physics, can reject the very method of approach and they will be right as well. It is due to the fact that economics studies human objects, the ones possessing reason and right to choose and making decisions, i.e. biological objects, while physics studies physical objects.

This principal difference (possessing reason and right to choose and making decisions) doesn't allow considering regularities and phenomena common to both types of objects. As for us, we are fundamentally examining the world of biological and physical objects from the position of their unity and we consider that biological and physical objects featuring both micro- and macro-level function according to the same laws (for example, principle of dissipation minimum of Oksanger-Prigogin-Moyseev). Oksanger and Prigogin formulated this principle for problems of physics (thermodynamics), and Moyseev - for problems of science studying

\footnotetext{
*National Academy of Science of Ukraine, Institute of the World Economy and International Relations, Civilization Problems Study Department, Leontovich str., 5, Kiev, 01030, Ukraine, Postal address: P.B. 4721, Dniepropetrovsk, 49094, Ukraine Tel.: +38 05 7771995 tel./fax: +380562929623e-mail: unid@a-teleport.com
} 
biological objects including economics (Moysseev, 1990). Therefore, we strictly believe that the break in the sphere of perception of the nature of economic dynamics and development will appear at the meeting-point of economics and non-linear physics. And we hope that this work will become if not a first step, then at least a considerable incentive for such a step.

\section{A little bit of history and formulation of the problem}

The problem of economic cycle, and necessity of theory explaining this problem, originated in $18^{\text {th }}-19^{\text {th }}$ centuries as a result of examining the crisis (and was mentioned in the works of C. Juglar; W. Jevons; J.Mills; T.R. Malthus; D. Ricardo). It was the necessity to withstand the economic crisis and its consequences, which led to the creation of the theory of cycle, economic dynamics and, as a result, anti-cyclic economic policy. The most complete fundamental work analyzing the achievements of the theory of economic cycles, economic development and economic dynamics is the work of A. Hansen "Economic cycles and national income" (1951). It started with "Analysis of the nature of economic cycles" (it is the title of the first chapter) and ended with conclusion "Unsolved problems" (subtitle of the Chapter 24, "Modern theory of cycle. Resume"). It is appropriate to quote him here: "Important elements (corner stones) of modern theory of economic cycle and authors who have greatly contributed to science can be listed in the following way:

1. Role of fluctuations of the investment volume (Tugan-Baranovsky, Spithoff, Gassel, Robertson).

2. Analysis of determinants of investment: natural rate taken in ratio to money rate of interest or - in more common terms - schedule of maximum effectiveness of investments to interest rate (Vicksel, Keynes).

3. Role of dynamic factors: equipment, natural resources, territory enlargement and growth of population as determinants of investment (Spithoff, Harrod).

4. Clustered nature of investment conditioned by gregarious nature of movement, due to activity in the sphere of innovations (Shcumpeter)

5. Capitalistic method of production (need in long period of time for production of elements of fixed capital, longevity of fixed capital) and principle of acceleration (Aftalion, Pigou, Klark).

6. Initial impulses and dissemination of cyclic movements conditioned by economy structure (Vicksel, Pigou).

7. Investment multiplier and consumption function (Kan-Keynes).

8. Interdependence of economic variables - economic models (Tinbergen, Frish, Samuelson, Hiks, Klein, et al.)". "During the whole history of literature about economic cycles, various economists again and again expressed their opinion that origin of cyclic fluctuation remains an unsolved riddle" (Hansen, 1997) Nevertheless, summarizing different types of economic cycles according to Kondratyev (short - 3-5 years, medium - up to 11 years and long cycles) has resulted in understanding the nature of cyclic recurrence. Different cycles have originally different causes without a common base. From our point of view, hypotheses of Gassel and Kondratyev are the most interesting. Gassel assumed that certain correlation exists between growth of population and amplitude of fluctuation of economic cycles (Gassel, 1932). Kondratyev advanced the presupposition ${ }^{1}$ that original reason initiating both economic dynamics and rise of cyclic recurrence in economy is growth of population during long periods of time, which results in two opposite trends: trend of increase in production and growth of consumption. Namely these two opposite processes are the consequences of growth of population and initiate economic dynamics and cyclic recurrence (Kondratyev, 1998). Later works in this sphere of economics have failed to produce considerable results in determining the nature of economic cycles' origin. So the problem is still open today.

Non-linear physics and wave process. Why is it imperative for economics? A few words about methods. Even in earlier works of J. Shcumpeter (1911), N. Kondratyev $(1925,1931)$ and Harrod (1948), it is stated that:

\footnotetext{
${ }^{1}$ N. Kondratyev was very close to formulating a general theory of social development. Being a prisoner in 1931, he managed to sketch "Main problems of economic statics and dynamics" (first publication in 1989). He also created a theory of economic trend. This work was lost in archives of security services of the USSR, though some chapters were published. Kondratyev tragically died in 1938.
} 
- statistical methods of classical mechanics came to economics from physics (Kondratyev, 1998; Schumpeter, 1982);

- achievements of statistical methods of economic theory based on the postulate of equilibrium state of ideal object have exhausted themselves for economic analysis. A need has emerged for methods of dynamic character and dynamic economics (Harrod, 1997).

But it was the linear method of analysis, which dominated that time. So, dynamic economic models were linear in essence. Further works of the second half of the $20^{\text {th }}$ century of Puankare, Thom and Uytny, Abraham and Shaw, Haken, Prigogin and Klymantovitch led to development of application of the theory of catastrophes, theory chaos, theory of non-equilibrium processes and self-organization. Understanding natural non-equilibrium of analyzed objects became the basis for such development. Non-equilibrium of single object and medium as a whole is a primary, initial, given by nature, condition of existence and development of the world. Before we consider the fluctuation of the object in space and in time as deviation from equilibrium state and nowadays, we will deal with chaos - forefather of all existence. Initial non-equilibrium of observed objects and occurrence of catastrophic disturbance, i.e. extremely non-equilibrium states leading to vagueness of further states of the objects and trajectory of development, resulted in creation of new methods of analysis: non-linear dynamic simulation. Haken and Prigogin aim at adapting of results of non-equilibrium thermodynamics to the sciences of life (Haken, 1996), but at the same time N. Moysseev and T. Puu examine such achievements in connection with problems of the general theory of development (N. Moysseev) and non-linear economic analysis (T. Puu) (Puu,2000; Moysseev, 1990).

N. Moysseev adapted Oksanger-Prigogin's principles of minimum dissipation to the problems of development of biological systems (Moysseev, 1990). T. Puu told about necessity of non-linear dynamic simulation in economics because linear dynamic simulation is significantly limited for solving economic problems. "The model of linear dynamics can describe either an explosion or a completely ceased motion" (Puu, 2000). Besides, Puu gave a summary of methods and literature for the problems of non-linear dynamics (Puu, 2000). They were the following:

- methods of disturbance (Stoker (1950); Jordan and Smith (1977); Kevorkian and Cole (1981)); Hayasi (1964);

- structural stability and catastrophe (Peixoto (1977); Hirch and Smale (1974); four-volume edition of Abraham and Shaw (1982); Poston and Steward (1978));

- chaos and fractal (Devaney (1986); Mandelbrot (1982); Peitgen and Richter (1986); Levi (1981). In summary, it is possible to define some order of evolution of physical methods. In classic mechanics, an analyzed object is, as a rule, single (taken out of context of the world) and fluctuation is an object's feature describing its deviation from the state of permanent equilibrium. Cyclic recurrence is a repeated deviation from an equilibrium state due to external disturbance. The next step in evolution of scientific thought - quantum physics - broadened the comprehension of fluctuation nature because it chose as an object not only a single object but the medium as a whole, i.e. a set of single objects. As a result, a phenomenon of the wave occurred. The nature of medium is the wave process, though single object is characterized by fluctuation. But simulation of wave processes used to be linear (for example, blast propagation of wave fluctuation). Further evolution had led to physics of non-equilibrium processes (for example, non-equilibrium thermodynamics), physics of open systems (Klymantovich, 1999) and nonlinear physics (Zaslavsky, and Sagdeev, 1988). Thus, today the main methodological problem of economics is that while recognizing non-equilibrium of phenomena, which theory of economic dynamics and economic development studies, we are trying to explain it by methods of linear dynamic simulation, which doesn't take into account the nature of non-equilibrium and occurrence of dynamics itself. Due to this, the problem of the nature of economic cycles is so imperative today.

\section{About non-linear physics}

According to the methodology, the course of non-linear physics first explains principles of behavior of fundamental particles and then of medium. As from the very beginning non-equilibrium is taken into consideration, i.e. uncertainty of a possible state of a particle in t point of time, so it is possible to methodologically determine particle location in space - phase-plane portrait (we don't mention phenomena of phase space and trajectory). In case of cyclic motion in space, when Hamiltonian system with one degree of free variable "action-angle" is 
introduced into phase-plane portrait, we face a phenomenon of invariant of motion, etc. It means that we have a set of categories and methods, which allows to approximately describing a complicated behavior.

Like with category of fluctuation when describing behavior of a single object, we have a category of wave process when dealing with medium. If for describing the state of particle in phase space we can use some finitedimensional vector $\mathbf{Z}=\mathbf{Z}(\mathbf{t})$, then for describing the medium it is necessary to introduce some quantity of fields FN $=\mathbf{F N}(\mathbf{r}, \mathbf{t})$, given in every point of space $\mathrm{r}$ at $\mathrm{t}$ moment of time. We would like to concentrate attention of readers on an ordinary circumstance: wave process appears when we are considering a medium. And one more common moment: wave is a process, a trend and not separate, systematically varying parameters of an object (this provides us with comprehension of cycle and cyclic recurrence in economics). Methods of description of wave processes are based on their own progression - from simple to complicated. In non-linear physics, problems of origin and evolution of waves are numerous and various enough. Therefore, the problem of travelling wave and origin of permanent front of wave is the most attractive for describing universal peculiarities of a wave process.

The first universality is availability of the process of wave torsion during motion of medium of non-interacting particles. The notion of particles' density at $\mathrm{x}$ point at $\mathrm{t}$ moment of time is also introduced: $\mathrm{n}(\mathrm{x}, \mathrm{t})$ (so-called non-linear term). Such characteristic of medium as density predetermines the motion of particles of front of the wave with different values of velocity. Values of velocity of various points of profile differ due to different density and, because of this, we can observe the process of torsion. This is further followed by indistinctness of profile, multiflow and destruction of profile). As a characteristic example, we can mention white horses on the top of sea wave at strong wind. Simple traveling wave with dispersing wave profile has one more name - Ryman's wave. Intensification of torsion leads to multiflow and umklapp process of wave profile. In reality umklapp process due to torsion doesn't happen every time. The reason is the medium, which possesses one more feature - viscosity, which creates a process of opposite direction - attenuation ${ }^{1}$. This phenomenon is described by Burgers's equation when a viscous term factor of viscosity $\mathrm{v}-$ is introduced into equation of motion of free medium:

$$
\frac{d v}{d t}+V \quad \frac{d}{d x}=0
$$

where: $\mathbf{V}$ - speed of medium; $\mathbf{v}=\mathbf{d x} / \mathbf{d t}$ - the function of point and time.

Then equation becomes:

$$
V t+V V x=v V x x
$$

Thus, one more universal feature of wave process is described - attenuation. If medium motion is considered together with non-linear property - density - and viscosity, then we can observe the third universal feature of wave process - permanent wave.

Permanent wave appears as a result of interaction of two processes of different directions: process of wave torsion $\downarrow$ and its attenuation $\uparrow$. Here we can formulate two interesting conclusions:

- Availability of two opposite trends generated by the properties of one object - medium - predetermines medium motion in the shape of permanent wave.

- In other words, under the influence of disturbing factor the state of medium becomes more stable: state of progress of wave process in the form of permanent wave.

Such a conclusion is very important for explaning the nature of wave process in economy and we will try to demonstrate this later. Here we only indicate that the most remarkable factor is that Burger's equation has exact solution. We are dealing with asymptotic solution of equation. It is already trivial for physicists. But it is a very important moment for economists. If we start from universality of the laws of motion of biological and physical mediums, then determination of utmost boundaries of wave profile can make possible description of utmost boundaries of wave fluctuations in economy and that is very important for defining the size of economic growth and crises. One more universal property of the wave process of the medium is $\mathrm{R}$ - Reynold's number. Dimensionless factor connecting ratio of non-linear and viscous terms is:

\footnotetext{
${ }^{1}$ Besides viscosity, there are some other properties, for example, dispersion.
} 


$$
R=\frac{V V_{X}}{V V_{X X}}
$$

Reynold's number is universal because it allows describing the utmost states of wave process. It is Reynold's number, which determines various modes of progress of physical processes. We will limit ourselves to the description of three universal regularities of non-linear wave processes: torsion, attenuation and, as a result of these two opposite processes, occurrence of permanent wave.

\section{From non-linear physics to economics}

As it has been already mentioned above, the main methodological problem of modern economics (to be more exact, its parts - theory of economic development and economic dynamics) is that, while recognizing nonequilibrium of economic objects, the problems of economic dynamics are solved by methods of linear dynamic simulation. The level of applied methods doesn't meet the complication of object analysis. Besides, the category of "economic medium" with one of its feature being wave process is absent from modern classic economic science (in all its directions). The absence of comprehension of aggregate of people and their associations as an economic medium makes it impossible to evaluate economic cycle as a wave process and, consequently, explain its nature. This happens because the classical condition in economics means that an individual has reason and, hence, can choose and make rational decisions. Since ordinary economic agent possesses reason mentioned above, microeconomy is guided only by linear dynamic models based on postulate of rationality and localization of economic interactions in time. However, namely the presence of reason and free will predetermines uncertainty of individual's behavior and, hence, chaotic behavior at microlevel. The method of reduction transfers rationality of behavior of one economic agent to the whole system and, at the same time, linear dynamic models are moved to macrolevel. However, the behavior of social systems at macrolevel is already of non-linear character. Uncertainty of behavior at microlevel forms non-linearity of behavior of macrostructure. The main problem lies in the fact that conscious behavior of a biological economic agent is not correlated with behavior of physical agents, which do not possess either reason or free will, due to the belief that biological and conscious nature and physical nature function according to different laws. This element of thinking is a stumbling block when analyzing the problems of economic dynamics. As we mentioned earlier, T. Puu greatly contributed to application of non-linear dynamic models for analysis of various types of cycles: trade cycles, business cycles (Puu, 1987), etc. He focused his attention on detection of dynamic chaos in models of economic cycles and availability of transitions from chaos to orderliness and back to chaos. This demonstrates non-linear properties of economic systems (Puu, 2000).

Here we would like to focus your attention on unity and universality of laws of development (motion) for biological and physical systems. As an example, we can mention conditions for occurrence of permanent wave process in physical and economic mediums.

We believe that any macroeconomic object can be designated as an economic medium and categories of phase space, trajectory, phase-plane portrait, etc. will be applicable to it. If we consider a separate person ordinary economic agent - as a field with definite coordinates at $\mathrm{x}$ point at $\mathrm{t}$ moment of time whose state can be expressed in simple ort in phase space,

$$
N=N(t)
$$

then economic medium can be described as an aggregate of fields given in every point of medium $\mathrm{R}$ at $\mathrm{t}$ moment of time.

$$
\mathrm{Fe}=\mathrm{Fe}(r, t)
$$

The process of economic development in long period of time can be described as motion of economic medium, which is brought into wave process that appears as a result of action of opposite processes initiated by the properties of this medium. What are the features of homo economicus which form the properties of the economic medium and the very wave process? 


\section{Homo average - ordinary field of economic medium}

If an ordinary economic agent - a human being - is considered as a subsystem, a part of a bigger system society, then it becomes clear that he possesses a series of various functions. The most important of them is biological, that is to retain vital activity of socium through continuation of species. Next is homeostasis. From cybernetic point of view, retaining of biological homeostasis (temperature of the body, humidity, weight, etc.) is the necessary condition for executing the function of continuation of species. From position of a system, the main function is function of development. Exactly through development biological homeostasis is retained because development means the process of accumulation of structural information complicating the structure of system (rules of conduct) and increasing its resistance to environment.

We understand economic development as the motion of economic medium given in sum of economic fields at $x$ point and at $t$ moment of time.

Thus, the main biological function of economic medium - continuation of species - is executed by a mechanism of retaining biological homeostasis through the process of motion of this medium. From the position of cybernetic approach a human being also possesses the property of feedback. Thus wave process in economic medium expressed by cyclic fluctuations is obligatory and necessary condition for initiating the process of accumulation of structural information, which, in its turn, is the basis for realization of main function of global socium (economic medium) - surviving of human race.

Turning back to prior formulated question about human features forming properties of economic medium, and processes forming permanent wave in this medium, we will underline the following. If the main system function in the process of development of ordinary economic agent is accumulation of structural information, decreasing its disorganization and increasing its resistance, then consumption becomes the dominating function retaining homeostasis and property of agent. It means consumption of biological food, clothes, shoes, heat, electric power, etc. But consumption is impossible without production of material benefits. So the second feature of homo economicus is his ability to work and to exchange his labour for benefits to consume or retain biological homeostasis. Thus, every ordinary field of economic medium executing its main function - continuation of species through mechanism of retaining biological homeostasis - demonstrates two main properties: production and consumption. On a scale of economic medium, these properties form two opposite processes: - process of production of benefits $\uparrow \cdot$ process of consumption of benefits $\downarrow$. In their turn these two opposite processes initiate the formation of permanent wave process, which is described by a simple equation of medium motion. In other words, equation of economic development can be expressed by equation of motion of economic medium given in set of fields.

Why and how does the wave appear? Or once more about the wave process in economic medium.

As we have mentioned earlier, wave processes studied in physics are based on the assumption that wave is a consequence of disturbance of medium by external source. For example, wave appears on the liquid surface due to impact of wind. It means that in observing and describing the wave, we deal with:

- medium or liquid;

- $\quad$ source of disturbance - wind;

- universal property of medium: density creating non-linear process of torsion of wave;

- universal property of medium: viscosity forming the umklapp process;

- universal property of medium: formation of permanent wave process as a more stable form of medium existence on the basis of the first two properties. So we state that medium exists, source of disturbance of medium exists, universal properties of medium - density and viscosity - are available, which under the influence of external source initiate two opposite processes: torsion and attenuation of wave. These processes acting against each other create a permanent front of wave, i.e. medium transfers from calm, continuous form of existence to wavy one. It transfers to the state, which is more resistant to influence of a source of disturbance (of environment). Physicists will find such details dull and elementary but they are necessary for understanding the essence of wave processes in economic medium. As we have already 
defined, in economy we also deal with economic medium, with two properties of medium - to produce and consume - two opposite processes of production and consumption. The main function of economic medium - socium - is retaining homeostasis. But we also know that human life has physical limit: 70-75 years on average. Man dies, and there is no necessity to maintain homeostasis anymore. So, the main function aimed to retain socium is continuation of species. Retaining homeostasis is a mechanism of realization of the main function only. How are functions of retaining of homeostasis and reproduction of species (or increase of population) connected with each other? If in socium - economic medium consisting of males and females able to increase population, some state of equilibrium in given limits homeostasis - has been achieved, then such socium will necessarily increase its population. To put it differently, having homeostasis (or equilibrium) retained in given limits will necessarily lead to growth of population!!! Namely at this point an economic dynamics as motion of economic medium appears. As we have demonstrated before, the motion of medium is described by a simple equation of motion of the type of Burgers's equation, which has exact solution. We can define utmost boundaries of cyclic economic fluctuations of wave process and this will allow us to understand both the size of economic growth and the depth of crisis in economy.

The growth of population leads to disturbance of medium in the form of increase of both production and consumption. It means that we face the functions of medium of production and consumption in time. These two processes of opposite directions create a simple permanent wave, which we can observe as an economic cycle expressed in various forms (industrial cycle, trade-and-industrial, technological, business cycle, etc.) depending on reasons which, to our opinion, initiate such economic cycle.

Under the pressing of population growth economic medium begins to conform to the shape of wave - more stable and complicated structural state.

\section{From chaos to order. Initiating economic ordering}

The growth of population is a factor disturbing economic medium (some economists will say that it has been already written by Malthus, Klarc, Kondratyev, Gassel, Harrod, Hansen, etc.). But the matter is that socium has one more factor disturbing the medium: limited resources. Growth of population leads to necessity to distribute available limited resources among new coming members of society. A child starting from the age of 8 consumes no less than any adult member of society. But resources for production and amount of produced benefits are limited in this society. And this fact is one of the most important principles of analysis in economics. If the given function of consumption increase is available as a consequence of continuos growth of population, then socium needs a mechanism of effective distribution of resources for production and benefits for consumption. Such a mechanism is society organization, i.e. a set of rules regulating the behavior of the members of the society. Society, in fact, appears from a group of people when they self-organize their own behavior through rules. Here we would like to digress from economic to physics. What is the difference between turbulent and laminar motion? It is in a more complicated form and, as a consequence, structure, as well as a greater resistance to the influence of external source increasing velocity of flow. It means that there are intervals in velocity where laminar form of medium existence is unstable and can transfer to more resistant form of existence - turbulent - with increased velocity of medium. As we can see, universality of laws of development - motion of economic medium - is applicable for physical medium. According to Prigogin and Haken, the process of self-organization creates a more complicated structure of the medium and it is realized in circumstances of extreme non-equilibrium state of medium which occurred due to extreme increase in motion velocity. In such a situation, the Oksanger-Prigogin's law of minimum of energy dissipation is realized. It means that at turbulent motion, the medium dissipates less energy if compared with laminar motion. To put it differently, medium creates less entropy - disorganization - and this make it more resistant to the influence of external factor, which increases velocity of medium motion. We can observe the same situation in economic medium and this confirms the universality, unity of laws of development of economic medium and motion of physical medium. Economic development is a form of motion of economic medium based on the same laws according to which physical medium moves and which are the subject of study of non-linear physics.

\section{And further to maximum boundaries of economic stability and self-organization}

As we know from physics, a turbulent form of medium motion is more stable if compared with laminar and it appears as a result of increase in velocity of medium motion. This example demonstrates that laminar form of 
medium motion being stable in certain interval of velocity can lose its stability in circumstances of increased velocity of motion. So, we can speak about availability of utmost boundaries of stability for one form of medium existence in some interval of motion velocity. It follows from this that a certain order of economic medium socium - exists for every form of motion of economic medium, rate of economic growth. This order has its own structure, adapting medium to population growth and keeping wave process in the given boundaries. And each order has its own boundaries of stability. As the wave process is defined by opposite trends of growth of production and consumption, it is reasonable to note that each mode of motion of economic medium will be defined by ratio of the function of consumption to the function of production, like Reynold's number. The ratio of the function of consumption to function of production is coefficient $K$ and it will be optimal if its value is close to $K \approx 1$ for the form of motion of the most stable economic growth. Value $\mathrm{K}<1$ or $\mathrm{K}>1$ defines the state of motion between most stable growth and its maximum and minimum possible forms and each form will have its own utmost value of ratio of production and consumption. In other words, this ratio will characterize utmost states of wave process of economic medium as well, i.e. will define boundaries of stability of socium with a given form of motion or a given rate of economic growth. Thus, having calculated boundaries of wave process of economic medium we will be able to determine those areas which correspond to occurrence of extremely non-equilibrium states (processes of transformation) under the influence of population growth in circumstances of limited benefits for consumption. To put it differently, organization of the given form of motion of economic medium fails to optimize distribution of limited recourses and, hence, its structure loses stability. The amplitude of economic fluctuation exceeds the bounds of a stable process. The indicator of consumption is quickly increased if compared with production. The value of coefficient $\mathrm{K}$ becomes $>>1$. The process of self-organization of economic medium - socium - comes into effect on the basis of motion of electorate majority while creating and realizing macroeconomic policy of power party (rules of economic behavior established for this period of time in socium) (Chistilin, 2001). In reality the process of self-organization is expressed by quick and smooth changing of direction of macroeconomic policy leading to change of socium structure - rules of economic game, and further to introducing the values of attenuation of wave process in economic medium in the frame of stable economic development expressed by value of $\mathrm{K}$ tending to one: $\mathrm{K} \rightarrow \mathrm{K} \approx 1$

\section{Conclusions}

1. A big open dissipating self-organizing economic system can be presented as an economic medium and be given in an aggregate of economic fields at $x$ point at $t$ moment of time: $\mathrm{Fe}=\mathrm{Fe}(\mathrm{x}, \mathrm{t})$.

2. Economic medium is featured by properties of production of benefits and their consumption. In circumstances of unlimited growth of population during long intervals of time and limited resources, these features form two processes of opposite direction: process of production $\uparrow$ and consumption $\downarrow$.

2.1. Both opposite processes form a simple permanent wave.

2.2. Processes of production and consumption have a universal analogue in physical medium: process of torsion $\downarrow$ and attenuation $\uparrow$.

2.3. The formation of simple permanent wave of economic medium has analogue in physical medium and characterized as universal law. Properties of economic and physical mediums initiating a wave process are of universal character as well. Thus, we can make a very important conclusion: wave process, present in economy in the form of economic cycle of any type, keeps socium in stable state and is the demonstration of universal law of development expressed in the form of keeping stable the state of biological and physical medium in the form of wave process under the influence of permanent external disturbance.

3. The process of economic development can be expressed in motion of economic medium under the effect of disturbing factor - population growth - and is described by a simple equation of medium motion of the type of Burgers's equation which has exact solution in the form of asymptotic approximation.

3.1. The solution of this equation will make possible to describe wave economic process and define close maximal and minimal boundaries of balanced motion of economic medium, i.e. boundaries of stable economic growth. 
3.2. The possibility of solving equation of motion of economic medium in the form of wave process will help to determine rates of economic growth and economic crisis for analyzing medium (system).

4. A characteristic parameter describing modes of development is value of $\mathrm{K}$, which is equal to the ratio of the quantitative expression of consumption to the analogue expression of production process.

4.1. In case of stable economic growth $\mathrm{K}$ will be around unity: $\mathrm{K} \approx 1$.

4.2. In case when $K<<1$ or $K \gg>1$, the amplitude of wave process of economic medium will exceed the boundaries of stability of economic growth.

4.3. As a result of action of the mechanism of self-organization in circumstances of extremely nonequilibrium medium, medium structure is reconstructed making interaction of ordinary economic agents more effective. In its turn more effective interaction puts values of amplitude of economic fluctuation of wave process into boundaries of stable economic growth.

\section{References}

1. Bar Yam, Y. (1997), Dynamics of Complex Systems. Adisson-Wesley.

2. Chistilin, D. (2001), "The Problems of Self-Organization in Economic Theory. Case of Transition Economy". Conference proceedings for Scientific NEWconference of top level. Italy. University of Salerno. September, 2001.

3. Gassel G. (1932), Theory of Social Economy. L., p. 646.

4. Haken, H. (1996), Synergetics as a Bridge Between the natural and Social Sciences. Evolution, Order, and Complexity. London, N.Y. Ruotledge.

5. Hansen, A. (1997), Economic Cycles and National Income. Moscow, Economica, v. 2. p. 298 .

6. Harrod, R. (1997), Theory of Economic Dynamics. Moscow, Economica, pp. 48 - 49.

7. Klymantovitch, Yu. (1999), Static Theory of Open Systems. Moscow, Yanus-K.

8. Kondretyev, N. (1998), Main Problems of Economic Statics and Dynamics Moscow, Economica, p. 134.

9. Moysseev, N. (1990), A Man and Noosphere. Moscow, Molodaya guardiya”, p. 42.

10. Puu.T. (1987), Complex Dynamics in Continuos Models of the Business Cycles. Lecture Notes in Economics and Mathematical Systems. Berlin, Springer-Verlag, pp. 227-293.

11. Puu T. (2001), Non-Linear Economic Dynamics. Izhevsk, Udmurt University.

12. Schumpeter, J. (1982), Theory of Economic Development. Moscow, Progress, p. 137.

13. Stengers, I., Prigogin, I. (1986), Order from Chaos Moscow, Progress.

14. Zaslavsky, G., Sagdeev. R. (1988), Introduction to Non-Linear Dynamics. From Pendulum to Turbulance and Chaos. Moscow, Nauka. 


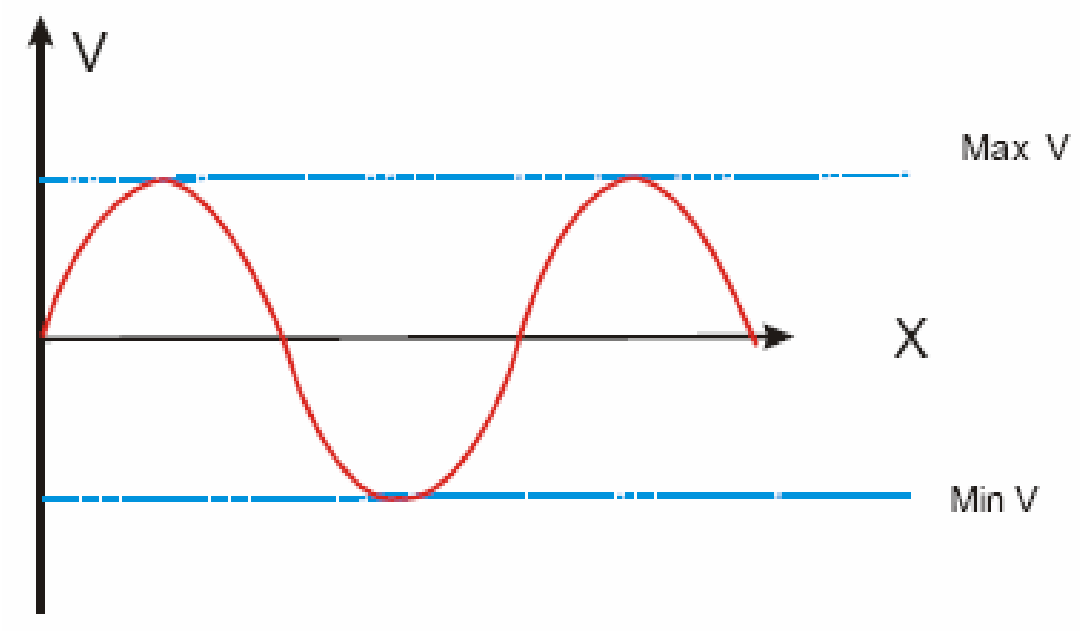

Figure 2. D. Chistilin

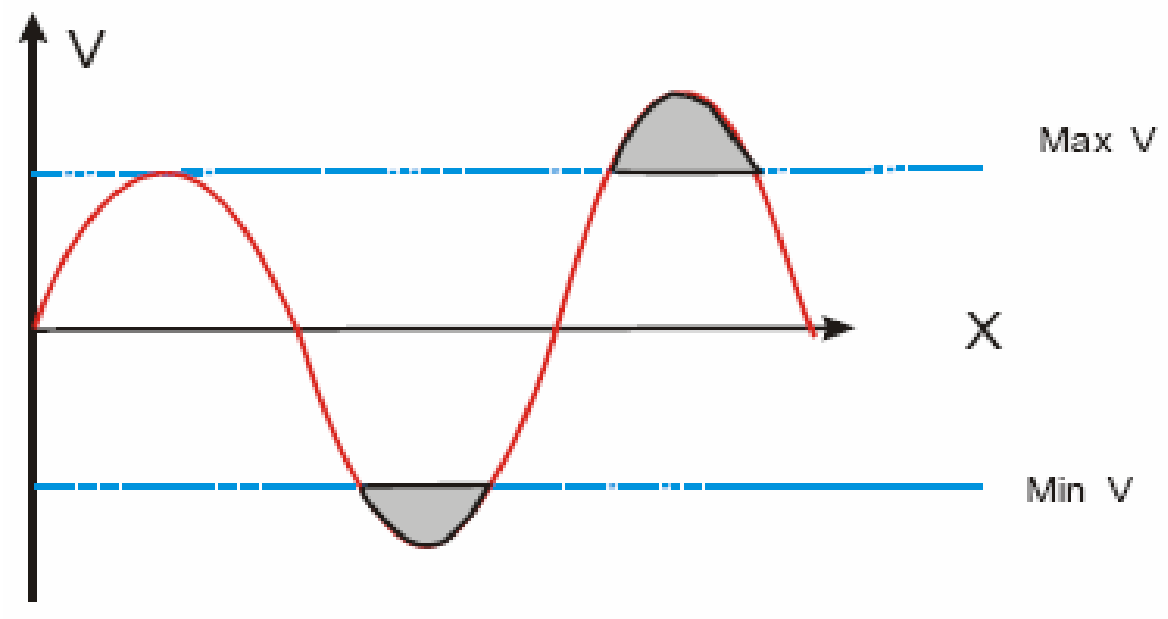


